\title{
Avaliação da Escala de Motivação Acadêmica em estudantes paulistas: propriedades psicométricas
}

\author{
Maria Cristina Rodrigues Azevedo Joly ${ }^{-}$- Universidade São Francisco, Itatiba, Brasil \\ Eli Andrade Rocha Prates - Centro Universitário Adventista de São Paulo, São Paulo, Brasil
}

\begin{abstract}
Resumo
A motivação de estudantes se tem destacado no contexto educacional como um construto importante em função do ensino eficaz e aprendizagem significativa. Nesse sentido, este estudo investigou as propriedades psicométricas de uma versão brasileira da Escala de Motivação Acadêmica (EMA) aplicada em 170 universitários paulistas, sendo 71\% do gênero feminino, com faixa etária de 18 a 25 anos, dos cursos de ciências biológicas, educação física, nutrição, pedagogia e psicologia. Constatou-se que os estudantes apresentaram média maior no fator motivação intrínseca e menor média para o fator motivação externa por recompensas sociais. Verificou-se diferença estatisticamente significativa na autopercepção da motivação para a universidade dos estudantes apenas em função do curso frequentado. Houve correlação dos escores da escala com o rendimento acadêmico. A precisão da escala aferida pelo alfa de Cronbach é muito boa. Os resultados referentes à validade e precisão obtidos atribuem características psicométricas à EMA para universitários paulistas.

Palavras-chave: Teoria da autodeterminação, Motivação, Psicometria, Ensino superior.
\end{abstract}

\section{Assessment of Academic Motivation Scale for students from São Paulo: psychometric properties}

\begin{abstract}
The students' motivation has been highlighted in the educational context as an important construct, as a function of effective teaching and meaningful learning. According with this concept, this study aimed to investigate the psychometric properties of a Brazilian version of the Academic Motivation Scale (EMA) applied to 170 university students from São Paulo, of which $71 \%$ female, aged 18 to 25 years in the courses of biological sciences, physical education, nutrition, psychology and pedagogy. The results showed that the students' best averaged score were in intrinsic motivation factor and the worst was in social reward external motivation factor. There were significant statistical differences in the students' self-perception of motivation to university only due to course taken. There was correlation between the factors' scale with the academic performance. The Cronbach's accuracy was very good. The validity and accuracy results give the psychometrics' characteristics to EMA.

Keywords: Self-regulation, Motivation, Psychometrics, Higher education.
\end{abstract}

Entender o papel da motivação para a aprendizagem dos estudantes é tarefa complexa. Isso porque ela está no interior da pessoa e não pode ser vista, só pode ser estudada mediante autorrelato ou observação de comportamento, sendo comum entre os profissionais do ensino, educadores e pesquisadores, a valorização do ambiente escolar como o fator mais importante na promoção da motivação e da aprendizagem (Getzels \& Thelen, 1960). No entanto, Guimarães e Bzuneck (2008) objetam uma análise tão superficial para um problema de tal magnitude, sem o respaldo empírico e teórico que o assunto demanda.

Dizem os autores acima que a motivação de estudantes se tem destacado no contexto educacional como um construto importante de se conhecer, em razão do ensino eficaz e aprendizagem significativa. Muitos professores frequentemente demonstram forte preocupação a respeito de como motivar os

\footnotetext{
${ }^{1}$ Endereço para correspondência:

Av. Alexandre Rodrigues Barbosa, 45 - Centro

13.251-900 - Itatiba-SP.

cristina.joly@saofrancisco.com.br
}

alunos que não demonstram interesse em aprender, apresentando resultados insatisfatórios de aprendizagem (Guimarães \& Bzuneck, 2008).

A par disso, sabe-se que a educação do ensino superior, ao longo dos anos, promove nos estudantes mudanças tanto em nível pessoal como cognitivo, afetivo, profissional e social, decorrentes da diversidade de experiências que compõem o leque de ensinamentos a serem aprendidos, sejam em atividades obrigatórias, sejam em atividades nãoobrigatórias, acadêmicas ou não-acadêmicas (Pascarella \& Terenzini, 2005). Nesse mesmo sentido, Almeida, Soares e Ferreira (1999) consideram que a busca do aprendizado teria como alvo o sucesso acadêmico, numa concepção muito mais ampla, envolvendo o desempenho cognitivo dos estudantes (raciocínio, conhecimentos, habilidades), o afetivo (crenças, valores, atitudes, autoconceito, motivações, satisfação) e o social (relações interpessoais).

Vê-se, então, com base no olhar acadêmico, a motivação como um conceito que abarca diferentes sentidos ou construtos significativos à aprendizagem, merecendo múltiplos estudos, como a revisão de Murphy e Alexander (2000) em 20 termos fundamentais que se vinculam às diferentes linhas de 
investigação nesse campo. Dentre estes, merece destaque a Teoria da Autodeterminação como uma das grandes linhas de pesquisa, partindo da postulação de que os seres humanos são dotados de propensão natural para o desenvolvimento saudável e autorregulação (Deci \& Ryan, 1985; 2000; 2004).

Deci e Ryan (1985), autores da Teoria da Autodeterminação, tradicionalmente diferenciavam a motivação humana em intrínseca e extrínseca. $\mathrm{Na}$ motivação intrínseca, o comportamento é motivado pelo prazer da atividade em si, ocorre livre de restrições ou pressões, tem origem em necessidades psicológicas de competência, autodeterminação e relacionamento. $\mathrm{Na}$ motivação extrínseca, em contraponto, o comportamento é motivado pela consequência, numa busca por um desfecho distinto para a ação, alcançando efeitos desejáveis ou evitando os indesejáveis. Nessa visão dicotômica, os piores resultados, em termos de autonomia, com efeitos mais restritos nos comportamentos, recaem sobre a motivação extrínseca; inversamente, a motivação intrínseca mostra os melhores resultados em aprendizagem, criatividade, desempenho, entre outros.

No entanto, os resultados de pesquisas empíricas, feitas pelos próprios autores da teoria, Deci e Ryan (1985; 1991), possibilitaram distinguir diversos estilos de regulação do comportamento humano, variando em virtude do nível de autonomia ou autodeterminação, ou seja, a própria motivação extrínseca pode ser, em certo grau, autodeterminada, superando, dessa forma, a dicotomia. Vansteenkiste, Lens e Deci (2006) concordam com essa abordagem, pois grande parte dos comportamentos é regulada por razões extrínsecas. No entanto, pode haver um grande envolvimento da pessoa, e os resultados serem semelhantes aos obtidos com atividades motivadas intrinsecamente. Desse modo, as palavras-chave são internalização das regulações externas, significando que quanto maior for o grau de autodeterminação do comportamento, melhor a qualidade da motivação.

Segundo a Teoria da Autodeterminação, é proposto um continuum de autodeterminação (Reeve, Deci \& Ryan, 2004) que diferencia seis estilos de motivação, que variam qualitativamente, dependendo do sucesso na internalização das regulações externas do comportamento. Inicia-se com o nível de desmotivação, caracterizado pela ausência da motivação ou do pensamento proativo. Após, apresenta-se a regulação externa como a forma mais básica e menos autônoma, quando o comportamento é controlado por recompensas e ameaças. Na sequência, como primeiro nível de internalização, propõe-se a regulação introjetada, cujos marcos regulatórios não são tão explícitos e a regulação é mais afetiva, envolvendo a resolução de impulsos conflituosos (fazer ou não fazer), e os comportamentos resultantes das pressões internas podem resultar em culpa e ansiedade (Pelletier, Fortier, Vallerand, Tuson, Brière \& Blais, 1995).

O estilo motivacional extrínseco por regulação identificada é verificado quando um comportamento é motivado pela apreciação dos resultados que produz. É mais autônomo do que os estilos de regulação apresentados anteriormente, mas ainda centra-se na consequência ou nos benefícios decorrentes.

Já o estilo motivacional por regulação integrada é a forma mais autônoma ou autodeterminada de regulação externa de um comportamento. Considera-se esse tipo volitivo, dada a possibilidade de escolha e importância para os objetivos pessoais de um indivíduo. Deci e Ryan (1985) sustentam que, apesar desse estilo de motivação extrínseca representar uma forma integrada e autodeterminada, considera-se comportamento motivacional extrínseco, já que é realizado visando à concretização de objetivos pessoais e não pelo prazer advindo do envolvimento nessa atividade.

Finalmente, na motivação intrínseca, a atividade é vista como um fim em si mesmo. Ryan e Deci (2000) reforçam ser o estilo perfeito de autodeterminação por envolver três componentes, que são o lócus interno (percepção de intenção e regulação pessoal), a liberdade psicológica (execução de um comportamento regulado pela vontade, interesse, necessidade e preferências da pessoa) e percepção de escolha (flexibilidade sobre o que fazer, como fazer ou não fazer).

No entanto, não se deve supor que um indivíduo siga necessariamente todas as fases do continuum para alcançar plenamente a regulação de um comportamento específico. Os estilos de regulação são referências para que se possibilite a análise do nível de internalização alcançado em um dado momento (Ryan \& Deci, 2000).

Nessa perspectiva teórica, diversas pesquisas têm descoberto relações entre os tipos de motivação extrínseca e a motivação intrínseca, com resultados bastante positivos para a aprendizagem (Grolnick \& Ryan, 1989; Lepper, Corpus \& Iyengar, 2005; Mayer, Faber \& Xu, 2007; Ryan \& Deci, 2000; Sheldon \& Kasser, 1998). No Brasil, verifica-se um interesse crescente por essa temática, sobretudo no ensino superior.

Destacam-se alguns estudos, como o de Guimarães e Bzuneck, em 2002, que levantaram as 
propriedades psicométricas de uma escala de avaliação da motivação (Work Preference Inventory, WPI), traduzida para o português e aplicada a uma amostra de 246 estudantes de licenciatura. Limitou-se a dois o número de fatores para as análises, motivação intrínseca e motivação extrínseca. A análise da consistência interna dos itens resultou em um alfa de Cronbach de 0,66 para o fator 1 (motivação extrínseca) e 0,57 para o fator 2 (motivação intrínseca), num escore total de 0,60, demonstrando homogeneidade entre os itens. Para cada subescala ou fator, foram encontrados dois outros fatores, cujos itens se aglutinaram, referindo-se à preferência por desafios e satisfação, na motivação intrínseca, e preferência por reconhecimento externo e compensação, na motivação extrínseca.

Um segundo estudo a se destacar teve como objetivo identificar as orientações motivacionais de alunos universitários, na Perspectiva de Tempo Futuro (Alcará \& Guimarães, 2007). Participaram 180 alunos de biblioteconomia. O índice de consistência interna da amostra foi de 0,81 . Os 7 itens avaliaram a valorização das atividades e as razões admitidas pelo aluno para a realização das tarefas (percepção de instrumentalidade). O índice de consistência da instrumentalidade foi de 0,84 .

As médias indicaram que esses alunos estabelecem e valorizam as metas de longo prazo. Já os resultados da percepção de instrumentalidade sugeriram que os alunos percebem a utilidade das atividades realizadas no presente para a sua atuação futura, sendo mais entusiasmados, dedicando mais esforço para dominar as tarefas e tendo comportamentos mais motivados do que aqueles que as consideram apenas como treinamento.

Um terceiro estudo poder-se-ia ainda destacar, por tratar-se da Escala de Motivação para Aprender de Universitários (Boruchovitch, 2008), que tem como objetivo medir as formas mais extremas de motivação, intrínseca e extrínseca. Foi aplicada numa amostra de 225 universitários. A análise fatorial apontou dois fatores, fator 1 , motivação intrínseca e fator 2, motivação extrínseca, com correlação moderada e significativa entre eles $(r=0,47 ; p=0,00)$, e os índices de consistência interna foram 0,86 para a Escala Total, 0,84 para o fator 1 e 0,76 para o fator 2 . A variância total explicada foi de $29,55 \%$.

Destaca a autora que, embora os dois fatores tenham aparecido de forma destacada na análise fatorial, parecem ter bem menos independência entre si, necessitando, então, de estudos sobre a natureza dessas inter-relações, para que um continuum motivacional possa explicá-las melhor. Há que se destacar, no entanto, que pelo menos essas duas formas de motivação coexistem no ser humano (Lepper e colaboradores, 2005; Ryan \& Deci, 2000b; Ryan \& Deci, 2000a).

Pode-se observar, diante das pesquisas apresentadas, como a enorme rigidez no trato dos dados forma o pano de fundo, pois isso faz com que as descobertas sejam reconhecidas pela confiança depositada nos instrumentos utilizados. Deve-se, com insistência, buscar a validade dos instrumentos de avaliação, integrando as múltiplas origens de evidências, extraídas em contextos e em momentos distintos (Fairchild, Horst, Finney \& Barron, 2005).

Levando isso em conta e também em relação à Teoria da Autodeterminação, Vallerand, Pelletier, Blais, Brière, Senécal e Vallières (1992) desenvolveram uma escala de avaliação, largamente usada na investigação de diversas frentes, a Escala de Motivação Acadêmica (EMA). As qualidades psicométricas dessa escala foram estudadas por alguns pesquisadores (Fairchild e colaboradores, 2005; Mullan \& Markland, 1997; Vallerand e colaboradores, 1992; 1993). Algumas inconsistências foram destacadas por Fairchild e colaboradores (2005), como correlações mais altas entre a regulação introjetada e a motivação intrínseca do que entre a regulação identificada e a motivação intrínseca. Adicionado a isso, não foi encontrada correlação negativa entre a desmotivação e a motivação intrínseca. Resultados parecidos foram encontrados no estudo de Cokley (2000).

Outro ponto interessante a ser examinado é a falta de itens para a avaliação da regulação integrada, considerado o estilo mais autodeterminado de motivação extrínseca. Vale ressaltar que, em estudo mais recente, Vansteenkiste e colaboradores (2006) apresentam a regulação identificada como o tipo mais autodeterminado, não justificando a mudança.

A escala original foi traduzida para o português por Sobral (2003), para um estudo acerca da motivação de estudantes do curso de medicina. A partir daí, Guimarães e Bzuneck (2008) iniciaram o processo de validação transcultural e consequente avaliação psicométrica da Escala de Motivação Acadêmica (EMA).

Para validação de conteúdo, utilizou-se a análise de juízes, e a versão final passou a conter 31 itens. Mediante o Método de Análise dos Componentes Principais, surgiram sete fatores (desmotivação, motivação extrínseca por regulação integrada, motivação extrínseca por regulação introjetada, motivação extrínseca por regulação externa de frequência às aulas, motivação extrínseca de regulação externa por recompensas sociais, motivação extrínseca por regulação identificada e 
motivação intrínseca) que explicaram $56,49 \%$ da variabilidade total dos dados.

Os índices de consistência interna de cada um dos sete fatores encontrados são considerados aceitáveis, avaliados pelo alfa de Cronbach, excetuando-se o índice de consistência para a Escala de Avaliação da Regulação Identificada. Na procura por evidências para a proposição de um continuum de desenvolvimento da autodeterminação, correlacionaram-se os resultados de desempenho dos participantes em cada avaliação. As correlações apoiaram a propositura de um continum de autodeterminação para os estilos de regulação do comportamento.

Conforme indicaram os autores (Guimarães \& Bzuneck, 2008), a EMA é um instrumento que necessita de revisões, a fim de melhor atender ao pressuposto teórico de que haja um continum de desenvolvimento da autodeterminação, principalmente no quesito de novos itens para a avaliação de alguns estilos de regulação, bem como, investigações com outras amostras da população.

Nesse sentido, a Escala de Motivação Acadêmica (Guimarães, Bzunec \& Joly, 2010) teve a continuidade de seu desenvolvimento, a partir de 2009, centrando-se em 1.000 estudantes universitários, sendo 61,5\% de duas Instituições de Ensino Particular (IES) paulistas e 38,5\% de uma Instituição Pública paranaense. Especificamente, o estudo com universitários paulistas caracterizou-se por analisar evidências de validade de construto e precisão da EMA, quando foram excluídos os itens 25 e 28, por não apresentarem carga fatorial condizente, passando a escala a ter 29 itens.

Levando-se os estudos anteriores em conta, o objetivo desta pesquisa foi analisar as características psicométricas da Escala de Motivação Acadêmica (EMA). A validade foi verificada por meio da correlação com desempenho acadêmico de universitários ingressantes e a precisão pelo alfa de Cronbach. Ao lado disso, foi caracterizada a autopercepção dos universitários quanto à sua motivação para a universidade, considerando as variáveis idade, gênero e curso frequentado.

\section{Método}

\section{Participantes}

A amostra foi composta por conveniência, constituindo-se de universitários brasileiros de Instituição de Ensino Superior (IES) paulista. Foram participantes deste estudo 170 universitários ingressantes, com idades entre 18 e 25 anos, sendo $29 \%$ do gênero masculino $(\mathrm{N}=49)$ e $71 \%$ do feminino $(\mathrm{N}=121)$. Dos participantes, $19 \%$ estavam regularmente matriculados no curso de ciências biológicas, $25 \%$ em educação física, em nutrição $16 \%$, pedagogia $16 \%$ e $24 \%$ em psicologia. Estes cursos se compuseram como pertencentes às áreas humanas $\mathrm{e}$ biológicas.

\section{Instrumentos}

Escala de Motivação Acadêmica - EMA (Guimarães, Bzunec \& Joly, 2010)

A EMA tem por objetivo caracterizar a autopercepção de estudantes universitários quanto à motivação para frequentar a universidade. É um instrumento de autorrelato, composto por 29 itens do tipo Likert com cinco pontos (1-discordo totalmente; 2-discordo muito; 3-nem discordo/nem concordo; 4concordo muito; 5-concordo totalmente) cuja pontuação pode variar de 29 a 145 pontos.

Todos os itens apresentaram carga fatorial e se distribuíram em seis fatores: desmotivação, motivação extrínseca por regulação introjetada, motivação extrínseca por regulação externa de frequência às aulas, motivação extrínseca de regulação externa por recompensas sociais, motivação extrínseca por regulação identificada e motivação intrínseca, explicados por $52,59 \%$ da variabilidade da escala.

A precisão foi aferida pelo coeficiente alfa de Cronbach, revelando-se adequada para a escala total, por apresentar o valor de 0,82. Esse resultado indicou uma fidedignidade satisfatória nos seis fatores da escala (desmotivação $=0,73$; motivação extrínseca por regulação introjetada $=0,75$; motivação extrínseca por regulação externa de frequência às aulas $=0,73$; motivação extrínseca de regulação externa por recompensas sociais $=0,64$; motivação extrínseca por regulação identificada $=0,54$ e motivação intrínseca $=0,81$ ).

Constatou-se ainda correlação significativa e direta entre os fatores, variando de 0,14 a 0,50 e inversa de 0,10 a 0,20 , exceção feita para o fator desmotivação com motivação extrínseca por regulação introjetada e identificada.

\section{Rendimento acadêmico}

Considera-se a média geral das disciplinas cursadas pelo participante, no semestre em que houve a coleta de dados. Essas médias foram compostas por 3 avaliações, por matéria cursada, sendo que houve, no mínimo, 2 instrumentos diferentes de avaliação, podendo um deles ser em grupo. Considerou-se também a nota obtida no vestibular de cada um dos participantes. Tanto as médias quanto as notas do 
vestibular foram fornecidas pela Secretaria de Registros Acadêmicos das IES.

\section{Procedimento}

Seguidos os protocolos éticos e as autorizações devidas, apresentou-se a pesquisa, e o objetivo foi informado aos estudantes, sendo-lhes assegurado o sigilo das informações e a liberdade de responderem ou não ao questionário. Em seguida, os participantes assinaram o Termo de Consentimento Livre e Esclarecido (TCLE) e responderam ao questionário, que foi aplicado coletivamente, em salas de aulas, com, no máximo, 80 estudantes. O tempo total de aplicação foi de aproximadamente 20 minutos.

\section{Resultados}

Primeiramente foram realizadas estatísticas descritivas da EMA para os universitários ingressantes. Para efeito de comparação entre os fatores da EMA, foi realizada uma média ponderada para cada fator, uma vez que havia um número variável de itens. $O$ cálculo foi realizado, considerando-se a média do fator dividida pelo número total de itens.

Observou-se que os estudantes apresentaram média maior no fator motivação intrínseca $(M=1,15)$ e menor média para o fator motivação externa por recompensas sociais $(M=0,19)$. A segunda maior pontuação deu-se para o fator motivação extrínseca por regulação introjetada $(M=0,56)$. Os outros dois fatores apresentaram-se com médias bem próximas, a saber, motivação extrínseca por regulação externa de frequência às aulas $(M=0,31)$ e motivação extrínseca por regulação identificada $(M=0,34)$.

As médias foram também verificadas por gênero e idade dos universitários. Com base nos resultados, o teste $\mathrm{T}$ de Student não revelou diferenças significativas para as duas variáveis. Posteriormente foram verificadas as médias dos fatores pelos cursos. Os resultados mostraram, de uma maneira geral, que as médias para cada fator foram similares entre os cursos.

Quando se realizou a ANOVA, para analisar as diferenças por curso, quanto à percepção da motivação para a universidade, as médias foram significativamente diferentes somente para os fatores motivação intrínseca $[F(4,165)=3,15 ; \quad p=0,01]$ e motivação por regulação identificada $[F(4,165)=2,47$; $p=0,04]$. Os outros fatores se apresentaram não significativos.

O teste Post Hoc Tukey (HSD) revelou diferenças no fator motivação por regulação identificada dos universitários de nutrição $(M=8,96)$ em relação aos que cursam psicologia $(M=10,75)$. Para o fator motivação intrínseca, o teste mostrou diferenças dos universitários de nutrição $(M=32,03)$ em relação aos que cursam pedagogia $(M=34,85)$. Os estudantes de educação física, ciências biológicas e psicologia apresentaram-se como pertencentes aos dois grupos.

Foi realizada também a correlação entre as notas dos universitários de cada curso e os fatores da EMA. Os resultados estão apresentados na Tabela 1.

Tabela 1. Correlação fator da EMA versus rendimento acadêmico dos estudantes em função do curso

\begin{tabular}{|c|c|c|c|c|c|c|c|c|c|c|}
\hline \multirow{3}{*}{ Cursos } & \multicolumn{10}{|c|}{ Notas } \\
\hline & \multicolumn{2}{|c|}{ Psicologia } & \multicolumn{2}{|c|}{ Pedagogia } & \multicolumn{2}{|c|}{$\begin{array}{c}\text { Ciências } \\
\text { Biológicas }\end{array}$} & \multicolumn{2}{|c|}{$\begin{array}{c}\text { Educação } \\
\text { Física }\end{array}$} & \multicolumn{2}{|c|}{ Nutrição } \\
\hline & $r$ & $p$ & $r$ & $p$ & $r$ & $p$ & $r$ & $p$ & $r$ & $p$ \\
\hline Desmotivação & $-0,04$ & 0,38 & 0,00 & 0,49 & $-0,33$ & 0,02 & 0,10 & 0,25 & $-0,29$ & 0,07 \\
\hline Introjetada & $-0,35$ & 0,01 & $-0,11$ & 0,29 & 0,12 & 0,24 & 0,01 & 0,45 & 0,31 & 0,06 \\
\hline Externa frequência & $-0,34$ & 0,01 & $-0,39$ & 0,02 & $-0,04$ & 0,41 & 0,11 & 0,24 & $-0,06$ & 0,38 \\
\hline Externa recompensa & $-0,17$ & 0,14 & 0,10 & 0,30 & 0,35 & 0,02 & $-0,01$ & 0,47 & $-0,04$ & 0,42 \\
\hline Identificada & $-0,34$ & 0,01 & $-0,09$ & 0,32 & $-0,08$ & 0,33 & 0,16 & 0,15 & $-0,01$ & 0,47 \\
\hline Intrínseca & $-0,16$ & 0,15 & 0,20 & 0,15 & 0,06 & 0,36 & 0,25 & 0,05 & 0,12 & 0,27 \\
\hline
\end{tabular}

Verifica-se que existe correlação positiva significativamente diferente de zero entre as notas dos universitários que cursam ciências biológicas e o fator motivação por regulação de recompensas sociais $(r=0,35 ; p=0,02)$, indicando que, quanto maior a motivação externa por recompensas sociais, maiores são as médias no curso. Houve ainda uma associação negativa e significativa entre o fator desmotivação e os estudantes de ciências biológicas, mostrando que, quanto menor a desmotivação, maior o desempenho.

Também há associações negativas significativamente diferentes de zero entre as notas dos 
estudantes de psicologia com os fatores de motivação por regulação introjetada $(r=-0,35 ; p=0,01)$, regulação externa de frequência às aulas $(r=-0,34 ; p=0,01)$ e regulação identificada $(r=-0,34 ; p=0,01)$, revelando que, quanto menor a motivação por regulação introjetada, por regulação externa de frequência às aulas $\mathrm{e}$ regulação identificada, maior o desempenho.

A Tabela 1 também revela existir correlação positiva significativamente diferente de zero entre as notas dos universitários que cursam pedagogia e o fator motivação por regulação externa de frequência às aulas $(r=0,39 ; p=0,02)$, indicando que, quanto maior a motivação por regulação externa de frequência às aulas, maiores são as médias no curso.
Quanto ao curso de educação física, há também correlação positiva significativa entre as notas dos estudantes e a motivação intrínseca $(r=0,25$; $p=0,05)$, indicando que, quanto maior a motivação intrínseca, maiores as médias no curso.

Em busca de evidências para a propositura de um continum de desenvolvimento da autodeterminação, correlacionaram-se os fatores. Como pode ser observado na Tabela 2, foram encontradas correlações positivas e significativas entre os itens que se aproximam no continuum, motivação introjetada, externa e identificada. A correlação negativa apareceu entre os itens opostos, desmotivação e motivação intrínseca e também motivação externa e intrínseca.

Tabela 2. Correlação entre os fatores da EMA

\begin{tabular}{|c|c|c|c|c|c|c|c|c|c|c|c|c|}
\hline \multirow[t]{2}{*}{ Fatores } & \multicolumn{2}{|c|}{ Desmotivação } & \multicolumn{2}{|c|}{ Introjetada } & \multicolumn{2}{|c|}{$\begin{array}{c}\text { Externa } \\
\text { frequência }\end{array}$} & \multicolumn{2}{|c|}{$\begin{array}{c}\text { Externa } \\
\text { recompensa }\end{array}$} & \multicolumn{2}{|c|}{ Identificada } & \multicolumn{2}{|c|}{ Intrínseca } \\
\hline & $r$ & $p$ & $r$ & $p$ & $r$ & $p$ & $r$ & $p$ & $r$ & $p$ & $r$ & $p$ \\
\hline Desmotivação & 1 & & 0,07 & 0,15 & 0,31 & 0,00 & 0,38 & 0,00 & 0,08 & 0,14 & $-0,23$ & 0,00 \\
\hline Introjetada & & & 1 & & 0,28 & 0,00 & 0,19 & 0,00 & 0,47 & 0,00 & 0,44 & 0,00 \\
\hline $\begin{array}{l}\text { Externa } \\
\text { frequência }\end{array}$ & & & & & 1 & & 0,26 & 0,00 & 0,37 & 0,00 & 0,12 & 0,05 \\
\hline $\begin{array}{l}\text { Externa } \\
\text { recompensa }\end{array}$ & & & & & & & 1 & & 0,09 & 0,11 & $-0,27$ & 0,00 \\
\hline Identificada & & & & & & & & & 1 & & $-0,50$ & 0,00 \\
\hline Intrínseca & & & & & & & & & & & 1 & \\
\hline
\end{tabular}

Os índices de consistência interna de cada um dos seis fatores, aferidos pelo coeficiente alfa de Cronbach, foram considerados adequados por apresentarem, para a escala total, o valor de 0,80 . Esse resultado mostrou fidedignidade aceitável nos 6 fatores da escala, desmotivação $=0,78$; motivação extrínseca por regulação introjetada $=0,71$; motivação extrínseca por regulação externa de frequência às aulas $=0,73$; motivação extrínseca de regulação externa por recompensas sociais $=0,60$; motivação extrínseca por regulação identificada $=0,66$ e motivação intrínseca $=0,65$.

\section{Discussão}

Um dos objetivos deste estudo foi verificar as propriedades psicométricas de uma versão brasileira da EMA (Guimarães, e colaboradores, 2010), já com adaptação de itens, utilizada, em sua versão original no Brasil com estudantes de medicina (Sobral, 2003). A validade foi verificada por meio da correlação com desempenho acadêmico e a precisão pelo alfa de
Crombach. Considerando-se ainda as variáveis idade, gênero e curso frequentado, outro objetivo foi verificar a autopercepção dos universitários acerca de sua motivação para a universidade.

Nos resultados coletados, observou-se que os estudantes, no geral, apresentaram média maior no fator motivação intrínseca $(M=1,15)$ e menor média para o fator motivação externa por recompensas sociais $(M=0,19)$. Isso revela, de acordo com a teoria de Deci e Ryan (1985), que o maior motivador do comportamento dos estudantes universitários, participantes do estudo, é o prazer pela atividade em si.

A segunda maior pontuação deu-se para o fator motivação extrínseca por regulação introjetada $(M=0,56)$, e os outros dois fatores se apresentaram com médias bem próximas, a saber, motivação extrínseca por regulação externa de frequência às aulas $(M=0,31)$ e motivação extrínseca por regulação identificada $(M=0,34)$. Esta conformação corrobora os estudos da EMA realizados por Guimarães, Bzuneck e Joly (2010) para as amostras de participantes paulistas e paranaenses. 
Estes resultados confirmaram o que propõe a Teoria da Autodeterminação (Deci \& Ryan, 2000), quando afirma não se poder explicar o desempenho pela dicotomia intrínseca versus extrínseca, já que, para os autores, existe uma tendência humana para a internalização dos valores e regulações do comportamento. A ideia, portanto, de um continum de desenvolvimento com base na regulação externa permite a visão de um comportamento autorregulado muito semelhante ao observado em situações de motivação intrínseca.

Sugere-se que, por estar a regulação introjetada como último nível de internalização nesta pesquisa, poder vir a ser interpretada numa posição no continuum mais adequada, próxima à intrínseca, considerando-se ainda, conforme Pelletier e colaboradores (1995), que os marcos regulatórios dela não são bem nítidos, pautando-se por uma regulação mais afetiva, promovendo a solução de impulsos conflituosos (fazer ou não fazer) que, de alguma forma, envolvem comportamentos resultantes de pressões interiores.

Em adição, os resultados mostram a regulação identificada ao lado da introjetada, como penúltima na internalização, o que também faz sentido. É um estilo bem autônomo, pois o comportamento é motivado pela apreciação dos resultados que produz. Portanto, também a necessidade do mundo interior do indivíduo se manifestar. Neste estudo, as regulações externas por recompensas sociais e frequência às aulas, portanto, tornaram-se, respectivamente, primeira e segunda motivações externas do continuum.

Essas mudanças no continuum, apresentadas na presente investigação, de alguma forma, trazem o questionamento, feito por Lepper e colaboradores (2005), se a motivação intrínseca e extrínseca são polos opostos de um mesmo construto ou se são construtos diferentes, independentes e/ou complementares, ou até mesmo, levando-se em conta as faixas etárias e escolares, ambas as alternativas possam fazer sentido.

É fato que os participantes deste estudo terem obtido maiores pontuações na avaliação da motivação extrínseca por regulação introjetada não confronta nem altera a hipótese teórica sustentada, atualmente, pela literatura, de que a introjeção seja uma forma de regulação menos autodeterminada do que a motivação extrínseca por regulação identificada. O que pode estar sendo apresentado, tanto nesta invetigação quanto em estudos anteriores, é a hipótese de que os itens utilizados não estejam refinados o suficiente para captar a sutil diferença entre eles, abrindo caminho para novas investigações.

Vale trazer também o estudo de Boruchovitch (2008) que, na análise fatorial de sua escala, encontrou os dois fatores, motivação extrínseca e intrínseca, bem definidos. No entanto, ao serem correlacionados, obtiveram correlação positiva moderada, explicando somente $29,55 \%$ da variabilidade da escala. Isso leva à reflexão de que ambas as formas de motivação coexistem no ser humano, mas há que se buscar empiricamente como este continum se define.

Quando se verificaram as médias por gênero e idade, perceberam-se similaridades, não se verificando diferenças significativas em todos os fatores. Essas similaridades também apareceram, de forma geral, quando se verificaram as médias dos fatores por curso.

O curso de nutrição apresentou maiores médias nos fatores de desmotivação e motivações extrínsecas, talvez ressaltando dificuldades em lidar com questões internas para a aprendizagem. Já o curso de psicologia apresentou maior média apenas no fator motivação por regulação identificada, isso podendo refletir o fato de que o estudo dos alunos é motivado pela apreciação dos resultados que eles esperam obter nos atendimentos que farão.

Interessante também é notar que o curso de pedagogia teve pontuação maior em relação aos outros somente em motivação intrínseca, levando-se à possibilidade de concluir-se que sejam alunos realmente motivados por uma causa, de acordo com a perspectiva de Getzels e Thelen (1960), bem como de Almeida e colaboradores (1999). Em posição inversa, por uma parte, e similar, por outra, apresentou-se o curso de educação física, obtendo as menores médias nos fatores desmotivação e motivação por regulação introjetada. Aqui surge uma aparente incongruência, pois este resultado faria mais sentido com a proposta do continuum a ser validada, já que a regulação introjetada aparece ao lado da desmotivação. Representaria, no caso, que o curso de educação física seja caracterizado pela motivação intrínseca e motivações extrínsecas também dependentes de alguma forma de interiorização (Deci \& Ryan, 1985, 1991) ou pela diversidade de atividades acadêmicas demandadas (Pascarella \& Terenzini, 2005). Tal resultado carece maior estudo.

Em sequência, ao proceder-se a realização da ANOVA, as médias foram significativamente diferentes exclusivamente para os fatores motivação intrínseca e motivação por regulação identificada. Os outros fatores se apresentaram não-significativos. O teste Post Hoc de Tukey para motivação intrínseca revelou diferenças somente para os universitários de nutrição $(M=8,96)$ em relação aos de psicologia $(M=19,75)$. Quanto à motivação por regulação identificada, o mesmo teste apontou diferenças somente entre os estudantes de nutrição $(M=32,03) \mathrm{em}$ relação aos que cursam pedagogia. Os outros cursos se apresentaram como pertencentes aos dois grupos. 
Há que se destacar a importância desta versão da EMA, justamente pelo fato de os autores terem elaborado novos itens para a motivação extrínseca, o que de pronto resolveu as inconsistências, destacadas por Fairchild, Horst, Finney e Barron (2005) para a versão original brasileira, como correlações mais altas entre a regulação introjetada e a motivação intrínseca do que entre a regulação identificada e a motivação intrínseca. Resolveu também o problema original da falta de correlação negativa entre a desmotivação e a motivação intrínseca.

Ainda em busca de evidências para a propositura de um continum de desenvolvimento da autodeterminação (Reeve e colaboradores, 2004), a correlação entre os fatores fortaleceu a EMA, pois correlações positivas e significativas foram encontradas entre os itens que se aproximam no continum (motivação externa, identificada e introjetada). Houve, além disso, correlação negativa entre os itens opostos, desmotivação e motivação intrínseca, como também entre motivação externa e intrínseca. Percebe-se que, nesta versão, as correlações indicam que o modelo teórico se confirma.

Fechando as análises deste estudo, a precisão da escala foi aferida pelo coeficiente alfa de Cronbach e considerada adequada por apresentar o valor de 0,80 . Esse resultado mostrou fidedignidade aceitável nos 6 fatores, variando de 0,65 a 0,78, assemelhando-se aos resultados de pesquisas anteriores (Fairchild e colaboradores, 2005; Guimarães \& Bzuneck, 2008; Guimarães e colaboradores, 2010; Vallerand e colaboradores, 1992; Vallerand, Pelletier, Blais, Brière, Senécal \& Vallières, 1993).

\section{Considerações finais}

Estudar a motivação dos estudantes para a aprendizagem é uma tarefa complexa, pois envolve um fenômeno de muitas facetas, que pode apenas ser inferido a partir de observação do comportamento ou pelo autorrelato. Muitas vezes, simplesmente a observação e as conclusões apressadas dão o norte para as atitudes do professor no processo ensinoaprendizagem (Getzels \& Thelen, 1960).

Isso, seguramente, não basta. Validar instrumentos para coleta de informações é imprescindível para que se possam conhecer os fenômenos psicológicos (Fairchild e colaboradores, 2005; Urbina, 2007). Conhecer os universitários brasileiros e seus estilos de regulação do comportamento é um passo importante para que se possa intervir no ambiente educacional, tornando os jovens autônomos, detentores realmente das decisões importantes de sua vida.
Nesse contexto, verifica-se a inserção da EMA. Um instrumento que já nasceu e se desenvolve gradativamente para ajudar a interpretar a motivação, construto complexo, importante, básico no processo da aprendizagem. Há questões a serem superadas, pois esta pesquisa tem caráter limitado, já que foi realizada numa população restrita, com número restrito de participantes também.

A hipótese da necessidade de refinamento dos itens que medem a motivação extrínseca por regulação introjetada e regulação identificada ofereceria ao pesquisador subsídios para a continuidade da investigação, buscando clarear a sutileza da diferença entre eles. A ampliação e diversificação da amostra também oportunizariam o aprofundamento da pesquisa sobre a temática.

\section{Referências}

Accorsi, D., Bzuneck, J. \& Guimarães, S. (2007). Envolvimento cognitivo de universitários em relação à motivação contextualizada. Avaliação Psicológica, 12(2), 291-300.

Alcará, A. \& Guimarães, S. (2007). Relações entre a perspectiva de tempo futuro e motivação de alunos universitários. Anais, VII Educere $V$ Encontro Nacional de Atendimento ao Escolar Hospitalar (pp. 1-8). Curitiba: Pontifícia Universidade Católica do Paraná - PUCPR.

Almeida, L., Soares, A. \& Ferreira, J. (1999). Adaptação, rendimento e desenvolvimento dos estudantes no ensino superior: construção / validação do Questionário de Vivências Acadêmicas. Braga: Universidade do Minho, Centro de Estudos em Educação e Psicologia.

Biddle, S., Markland, D., Gilbourne, D., Chatzisarantis, N. \& Sparkes, A. (2001). Research methods in sport and exercise psychology: quantitative and qualitative issues. Journal of Sports Sciences, 19, 777 809.

Boruchovitch, E. (2008). Escala de Motivação para Aprender de Universitários (EMA-U): propriedades psicométricas. Avaliação Psicológica, 7(2), 127-134.

Bueno, J., Zenorini, R., Santos, A., Matumoto, A. \& Buchatsky, J. (2007). Investigação das propriedades psicométricas de uma Escala de Metas de Realização. Estudos de Psicologia, 24(1), 7987.

Bzuneck, J. (2005). A motivação dos alunos em cursos superiores. Em M. Joly, A. Santos, \& F. Sisto, 
Questões do Cotidiano Universitário (pp. 217-237). São Paulo: Casa do Psicólogo.

Cockley, K. (2000). Examining the validity of the Academic Motivation Scale by comparing Scale Construction to Self-determination Theory. Psychological Reports, 86, 560-564.

Deci, E. \& Ryan, R. (1985). Intrinsic motivation and selfdetermination in buman behavior. Nova Iorque: Plenum Press.

Deci, E. \& Ryan, R. (1991). A motivational approach to self: integration in personality. (R. Dienstbier, Ed.) Nebraska Symposium on Motivation: Perspectives in Motivation, 38, 237-288.

Deci, E. \& Ryan, R. M. (2000). The "what" and "why" of goal pursuits: human needs and selfdetermination of behavior. Psychological Inquiry, 11(4), 227-268.

Deci, E. \& Ryan, R. (2004). Handbook of SelfDetermination Research. Nova Iorque: The University Rochester Press.

Fairchild, A., Horst, S., Finney, S. \& Barron, K. (2005). Evaluating existing and new validity evidence for the Academic Motivation Scale. Contemporary Edicational Psychology, 30, 331-358.

Getzels, J. \& Thelen, H. (1960). The classroom group as a unique social system. Em N. Henry (Ed.). The dynamics of instructional groups, sociopsychological aspects of teaching and learning: The 59th yearbook of the National Society for Study of Education. Chicago: University of Chicago Press.

Grolnick, W. \& Ryan, R. (1989). Parent styles associated with children's self-regulation and competence in school. Journal of Educational Psychology, 81, 143-154.

Guimarães, S. E. \& Bzuneck, J. A. (31 de março de 2008). Propriedades psicométricas de um instrumento para avaliação da motivação de universitários. Ciências \& Cognição, 13(1), 101-113.

Guimarães, S. \& Bzuneck, J. (janeiro-junho de 2002). Propriedades psicométricas de uma medida de avaliação da motivação intrínseca e extrínseca: um estudo exploratório. Psico-USF, 7(1), 01-08.

Guimarães, S., Bzuneck, J. \& Joly, M. (2010). Escala de Avaliação da Motivação para Universidade (AMU). Manuscrito não publicado. Universidade Estadual de Londrina / Universidade São Francisco.

Kline, P. (1994). An easy guide to factor analysis. Nova Iorque: Routledge.
Lepper, M., Corpus, J. \& Iyengar, S. (2005). Intrinsic and extrinsic motivation in the classroom: age differences and academic correlates. Journal of Educational Psychology, 97(2), 184-196.

Mayer, J., Faber, M. \& Xu, X. (2007). Seventy-five years of motivation measures. Motivation and Emotion, 31, 83-103.

Mullan, E. \& Markland, D. I. (1997). A graded conceptualisation of self-determination in the regulation of exercise behavior: development of a measure using conWrmatory factor analytic procedures. Personality and Individual Diferences, 23, 745-752.

Murphy, P. \& Alexander, P. (2000). A motivated exploration of motivation terminology. Contemporary Educational Psychology, (25), 3-53.

Pascarella, E. \& Terenzini, P. (2005). How college affects students: a third decade of research (2nd ed ed.). São Francisco: Jossey-Bass.

Pelletier, L., Fortier, M., Vallerand, R., Tuson, K., Brière, N. \& Blais, M. (1995). Toward a new measure of intrinsic motivation, extrinsic motivation and amotivation in sports: the Sport Motivation Scale (SMS). Journal of Sport \& Exercise Psychology, 17, 35-53.

Reeve, J., Deci, E. \& Ryan, R. (2004). Selfdetermination theory. A dialectical framework for understand sociocultural influences on student motivation. Em D. McInerney \& S. Van Etten (Orgs.). Big Theories Revisited (pp. 31-58). Connecticut: Age Publishing.

Ryan, R. \& Deci, E. (2000a). Intrinsic and extrinsic motivations: classic definitions and new directions. Contemporary Educational Psychology, 25, 54-67.

Ryan, R., \& Deci, E. (2000b). Self-Determination Theory and the facilitation of intrinsic motivation, social development, and well-being. American Psychologist, 55, 68-78.

Sheldon, K. \& Kasser, T. (1998). Pursuing personal goals: skills enable progress, but not all progress is beneficial. Personality and Social Psychology Bulletin, 24, 1319-1331.

Sobral, D. (2003). Motivação do aprendiz de medicina: uso da Escala de Motivação Acadêmica. Psicologia: Teoria e Pesquisa, 19(1), 25-31.

Urbina, S. (2007). Fundamentos da Testagem Psicológica. Porto Alegre: Artmed. 
Vallerand, R., Pelletier, L., Blais, M., Brière, N., Senécal, C. \& Vallières, E. (1992). The Academic Motivation Scale: a measure of intrinsic, extrinsic, and amotivation in education. Educational and Psychological Measurement, 52, 1003-1017.

Vallerand, R., Pelletier, L., Blais, M., Brière, N., Senécal, C. \& Vallières, E. (1993). On the assessment of intrincis, extrinsic, and amotivation in education: evidence on the concurrent and construct validity of the Academic Motivation Scale. Educational and Psychological Measurement, 53, 159-172.
Vansteenkiste, M., Lens, W. \& Deci, E. (2006). Intrinsic versus extrinsic goal contentes in SelfDetermination Theory: another look at the quality of academic motivation. Educational Psychologist, 41, 19-31.

Zenorini, R., Santos, A. \& Bueno, J. (2003). Escala de Avaliação das Metas de Realização: estudo preliminar. Avaliação Psicológica, 2(2), 165-173.

Recebido em 12/12/2010 Reformulado em 07/04/2011 Aprovado em 20/05/2011

Sobre os autores:

Maria Cristina Rodrigues Azevedo Joly é doutora em Psicologia pela Universidade de São Paulo, com pósdoutoramento em Avaliação Psicológica na Universidade do Minho, Portugal. É professora na graduação e na PósGraduação Stricto Sensu em Psicologia na Universidade São Francisco.

Eli Andrade Rocha Prates é graduado em Letras pela Universidade do Grande Rio (UNIGRANRIO), mestre em Psicologia pela Pontifícia Universidade Católica de Campinas (PUCCAMP) e doutorando em Psicologia pela Universidade São Francisco (USF). Atualmente é docente e coordenador de extensão do Centro Universitário Adventista de São Paulo (UNASP). Dentre os temas de interesse destaca-se a Avaliação Psicológica. 